\title{
Hotline sessions presented at the American College of Cardiology Congress 2009
}

\author{
Lars S. Maier · Magnus Baumhäkel • \\ Michael Böhm
}

Received: 15 April 2009/Accepted: 21 April 2009/Published online: 9 May 2009

(C) Springer-Verlag 2009

\begin{abstract}
The article summarizes the results of clinical trials in the field of cardiovascular medicine, which were presented during the Hotline Sessions at the annual meeting of the American College of Cardiology in Orlando, USA, from 28th March to 31st March 2009. The data were presented by leading experts in the field with relevant positions within the trials. Unpublished reports should be considered as preliminary data as the analysis may change in the final publications. The summaries presented in the manuscript were generated from the oral presentations and provide the readers with the comprehensive information on the results of the latest clinical trials in cardiovascular medicine.
\end{abstract}

Keywords NAPLES II · ARMYDA-RECAPTURE · ABOARD · OMEGA · REVIVAL-3 - EARLY-ACS · TIPS · ENCORE · AURORA · JUPITER - STICH · FIX-HF-5 · Pre-RELAX-AHF · PRIMA . HORIZONS-AMI · EPROTIROME · Heinz Nixdorf recall study · ACTIVE-A - REVERSE · Hypoxia-inducible factor- $1 \alpha \cdot$ ADMIRE-HF

L. S. Maier

Department of Cardiology and Pneumology, Heart Center, Georg-August-University Göttingen, Göttingen, Germany

M. Baumhäkel $(\bowtie) \cdot$ M. Böhm

Klinik für Innere Medizin III, Kardiologie, Angiologie und Internistische Intensivmedizin, Universitätsklinikum des Saarlandes, Kirrbergerstr., 66421 Homburg/Saar, Germany e-mail: magnus@baumhaekel.de
NAPLES II (novel approaches for preventing or limiting event study impact of a single high loading dose of atorvastatin on periprocedural myocardial infarction)

The purpose of the NAPLES II study was to assess whether a single, high $(80 \mathrm{mg}$ ), loading (within $24 \mathrm{~h}$ ) dose of atorvastatin is effective in preventing elevation of biomarkers of myocardial infarction (MI) following elective coronary stent implantation. Periprocedural MI was defined as an elevation of $\mathrm{CKMB} \geq 3$-fold of the upper limit of normal (non-Q wave $\mathrm{MI}$ ) or $\mathrm{CKMB} \geq 2$-fold of the upper limit with new significant $\mathrm{Q}$ waves in $\geq 2$ contiguous leads (Q wave MI) within $12 \mathrm{~h}$ after intervention. It was a prospective, randomized, double-arm, two-center clinical study in Italy. Altogether, 668 patients were randomized with 338 patients being assigned to the treatment arm, if they were not treated with a statin previously (mean LDL similar in both groups $126-129 \mathrm{mg} / \mathrm{dl}$ ). The results showed that atorvastatin reduced the incidence of periprocedural non-Q wave MI in elective PCI. This cardioprotective effect seems to be more pronounced in patients with high CRP level at baseline. Together these results suggest that the beneficial effects of atorvastatin may result from pleiotropic anti-inflammatory effects.

\section{ARMYDA-RECAPTURE (atorvastatin for reduction of myocardial damage during angioplasty)}

This study was a prospective, multicenter, randomized, double-blind trial investigating the efficacy of atorvastatin reload in patients on chronic statin therapy undergoing PCI. The primary endpoint was the 30-day incidence of cardiac death, MI, or target vessel revascularization (TVR). 


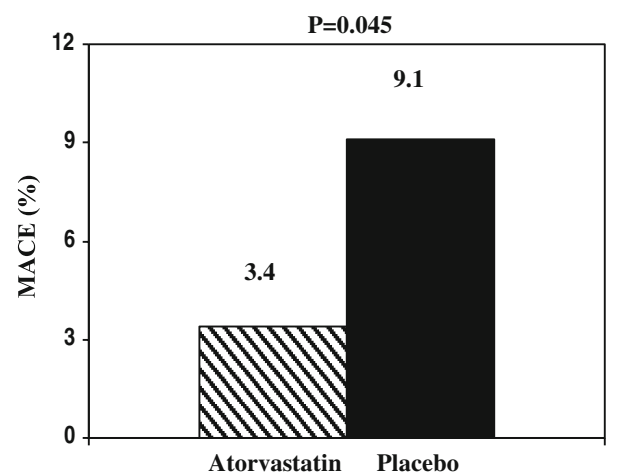

Fig. 1 ARMYDA-RECAPTURE: atorvastatin reduced the primary endpoint of 30-day MACE significantly

Patients with chronic ( $>30$ days) statin therapy and stable angina or NSTE-ACS undergoing coronary angiography were included in this study (mean LDL similar in both placebo and verum groups with $92-93 \mathrm{mg} / \mathrm{dl}$ ). Acute atorvastatin bolus $80 \mathrm{mg}+40 \mathrm{mg} 12 \mathrm{~h}$ pre-PCI was given in 177 patients (vs. placebo, $n=175$ ) which resulted in a significant $48 \%$ relative risk reduction of the composite primary end point at 30 days (MACE criteria) with a number needed to treat (NNT) of 17 (Fig. 1). The benefit was largely localized to patients who presented with ACS (with an $87 \%$ risk reduction, NNT $=9$ ). In addition, the incidence of postprocedural CK-MB and troponin elevation was significantly reduced. The study indicates that reloading with a high dose of atorvastatin was associated with improved clinical outcome in patients on chronic statin therapy undergoing PCI. Similar to NAPLES II, rapid LDL-independent cardioprotective effects may be responsible of this phenomenon. These findings may support a strategy of routine reload with high dose atorvastatin early before intervention even in the background of chronic statin therapy.

\section{ABOARD (angioplasty to blunt the rise of troponin in acute coronary syndromes randomized for an immediate or delayed intervention)}

The ABOARD study was a multicenter randomized trial to investigate whether immediate intervention (primary PCI strategy) is superior to delayed intervention (next day strategy) in patients with moderate to high-risk (TIMI score $\geq 3$ ) in 352 patients with NSTEMI-ACS. All patients were on optimal medical therapy with high percentages of aspirin, clopidogrel, heparin, ACE-inhibitors, $\beta$-blockers, statins. $65 \%$ of the patients in both groups additionally received abciximab. The time to catheterization (radial access in $84 \%$ of the patients) was $\sim 1 \mathrm{~h}$ for the immediate group $(n=175)$ and $\sim 20 \mathrm{~h}(n=177)$ for the delayed group. With respect to clinical endpoints (e.g. MI, death) as well as with respect to surrogate parameters (e.g. troponin, CK-MB) there was no difference between both groups.

OMEGA (randomized trial of omega-3 fatty acids on top of modern therapy after acute myocardial infarction: the OMEGA-trial)

Omega-3 fatty acids were recommended after myocardial infarction [10]. In this trial, $1 \mathrm{~g}$ omega-3 fatty acids where tested versus placebo (olive oil) on top of optimal medical therapy. The primary endpoint was sudden cardiac death. More than 3,800 patients 3-14 days after acute myocardial infarction (STEMI or NSTEMI) were included. A sudden cardiac death rate of $3.5 \%$ was assumed which may have been the reason that no differences were found between both groups having only $1.5 \%$ sudden cardiac death on average. However, even in the secondary endpoints (total death, reinfarction, stroke, progression of coronary artery disease, arrhythmic events) no changes were found. In contrast to the GISSI-HF findings (in patients with chronic heart failure) after 4 years of following-up the patients (published last year) 1-year follow-up may not have been enough to find differences between treatment groups in the present study investigating omega- 3 fatty acids in patients with MI.

REVIVAL-3 (prospective, randomized, double-blind, placebo-controlled trial of erythropoietin in patients with ST-segment elevation myocardial infarction undergoing percutaneous coronary intervention)

The objective of this study was to assess the effect of highdose erythropoietin in patients with acute ST-elevation myocardial infarction and $\mathrm{EF}<50 \%$ treated with primary PCI. 138 patients were assigned to two groups. The verum group received erythropoietin beta $\left(3.3 \times 10^{4} \mathrm{U}\right)$ at the time of balloon inflation, and after 24 as well as $48 \mathrm{~h}$. There was no difference in the primary end point of left ventricular EF after 6 months between both groups. Also infarct size was not reduced. Most disappointingly, there was even a trend towards a higher risk of adverse clinical events (MACCE at 6 months), which should be taken into account before planning future investigations with this drug in patients with acute MI. 
EARLY-ACS (early glycoprotein IIb/IIIa inhibition in non-ST-segment elevation acute coronary syndrome: a randomized, double-blind, placebo-controlled trial evaluating the clinical benefits of early front-loaded eptifibatide in the treatment of patients with non-ST-segment elevation acute coronary syndromes)

The goal of the study was to compare the effect of two strategies for eptifibatide administration in high-risk NSTE ACS patients managed with an invasive diagnostic assessment. The first strategy was a routine, early administration of eptifibatide to all patients shortly after presentation. The second strategy consisted of a delayed, provisional eptifibatide administration at the physician's discretion after coronary angiography and prior to PCI. In all five continents $>9,400$ patients were assigned to this study. Early eptifibatide compared with delayed, provisional eptifibatide at PCI did not significantly reduce the primary composite of death, MI, recurrent ischemia requiring urgent revascularization, or thrombotic bailout at $96 \mathrm{~h}$, but resulted in a trend toward reduction in death or $\mathrm{MI}$ at 30 days with no difference in 30-day mortality. Also, it resulted in higher rates of non-life-threatening bleeding and transfusions. Although the results of EARLY-ACS do not support a strategy of routine early eptifibatide use in high-risk NSTE ACS patients managed with an invasive strategy it is possible that a subgroup of patients who have a low risk of bleeding may be considered for early eptifibatide use [4].

\section{TIPS (The Indian Polycap Study)}

This randomized and double blind study investigated the effects of a polypill (Polycap) containing three blood pressure lowering drugs at low doses (thiazide $12.5 \mathrm{mg}$, atenolol $50 \mathrm{mg}$, ramipril $5 \mathrm{mg}$ ) with and without aspirin (100 mg) and simvastatin $(20 \mathrm{mg}) .400$ patients with at least one cardiovascular risk factor in India were randomized to this group and 200 patients were assigned to eight other groups (1: aspirin, 2: thiazide, 3: thiazide + ramipril, 4: thiazide + atenolol, 5: ramipril + atenolol, 6: thiazide + ramipril + atenolol, 7 : thiazide + ramipril + atenolol + aspirin, 8: simvastatin). Mean heart rate before the study was $80 \mathrm{bpm}$, systolic RR was $134 \mathrm{mmHg}$, and LDL was $3.0 \mathrm{mmol} / \mathrm{l}$. After 12 weeks the results clearly showed that RR was lowered linearly when increasing the number of RR lowering drugs up to $7 \mathrm{mmHg}$, heart rate was reduced in all atenolol groups by $7 \mathrm{bpm}$. LDL was reduced similarly in the simvastatin formulations compared to the Polycap group between 0.7 and $0.83 \mathrm{mmol} / \mathrm{l}$. It was estimated that the combination of all effects tested using the Polycap could reduce the relative risk for CHD by $62 \%$ and for stroke by $48 \%$. It is concluded by the authors that in patients with average risk factor levels, the Polycap consisting of five different cardiovascular drugs has similar effects than the combination of each of the single drugs, it is well tolerated, and most importantly it may potentially reduce CVD risk by about half [1].

ENCORE (cardiovascular benefits associated with the DASH diet alone and in combination with exercise and weight reduction in men and women with high blood pressure)

Cardiovascular risk is influenced by changes in habits [13]. The objective of this study was to examine the effects of (1) the DASH diet (1,962 kcal) and (2) the DASH diet plus exercise and weight loss $(1,648 \mathrm{kcal})$ on blood pressure in overweight patients with high blood pressure. About 150 patients were included in the study. The study showed that the DASH diet alone resulted in significant blood pressure lowering (by $11 \mathrm{mmHg}$ systolic blood pressure). However, exercise and weight loss in addition to the DASH diet resulted in an incremental decrease in blood pressure (by $16 \mathrm{mmHg}$ systolic blood pressure), as well as greater improvements in cardiovascular manifestations of high blood pressure.

\section{Home health monitoring reduces cardiovascular disease risk in medically underserved communities}

The objective was to lower cardiovascular disease risk in urban and rural underserved communities and to compare a nurse management program (four visits in 1 year) versus nurse management plus weekly reporting of CVD risk factors via telemedicine. Almost 400 subjects without CVD but an increased were included and divided into the two arms. The primary end point was a reduction in $5 \%$ or greater in CVD risk at 1 year. Although this endpoint was not reached, at least blood pressure was reduced to a greater extent in the telemedicine group. As compared to Caucasians, African Americans showed less blood pressure and lipid control. The authors concluded that a nurse management program can reduce CVD risk in medically underserved communities. Telemedicine provides additional benefit for blood pressure management. Male and female subjects achieved similar improvements in blood pressure and lipids. White subjects achieve lower blood pressure and lipid values compared to African Americans. 
AURORA (effect of rosuvastatin versus placebo on cardiovascular outcomes in patients with end-stage renal disease on hemodialysis)

This study showed that initiation of rosuvastatin did not cause a reduction in the combined endpoint of $\mathrm{CV}$ death, MI or stroke, even though LDL-C was significantly reduced and even a minor reduction in Hs-CRP occurred. Rosuvastatin treatment was well tolerated. The authors concluded that a lack of $\mathrm{CV}$ benefit with statins in both AURORA and 4D suggests that CVD in hemodialysis patients is different compared with that in a non-renal population. There is a need for further research and analysis of data and to explore new approaches and treatment strategies for reduction of the high risk of CVD in hemodialysis patients as stated in the published article by the authors the same day [3].

\section{JUPITER (CRP reduction, LDL reduction, and cardiovascular event rates after initiation of rosuvastatin)}

To answer the question, whether the effects of the JUPITER trial (20 mg rosuvastatin in healthy subjects) for the Prevention of Cardiovascular Events published last year (44\% risk reduction) where due to a reduction of LDL or CRP, this analysis was presented (Table 1). Among apparently healthy men and women initiating rosuvastatin therapy in the JUPITER trial, achieving low target levels of both LDLC and hsCRP was associated with significantly improved event-free survival compared with achieving neither target or with achieving a low LDLC alone. Similar effects were observed after adjustment for all available baseline clinical characteristics including entry levels of LDLC and hsCRP, and in analyses based upon ApoB or the ApoB:ApoA ratio rather than upon LDLC. A 79\% reduction in risk was observed among those who achieved LDLC $<70 \mathrm{mg} / \mathrm{dl}$ and the even more aggressive target of hsCRP $<1 \mathrm{mg} / 1$ [3].

\section{STICH: surgical treatment for ischemic heart failure trial-CABG versus CABG + SVR - hypothesis 2}

In the STICH-trial, 1,000 patients with ischemic heart failure $(\mathrm{EF} \leq 35 \%)$ and regional anterior-apical left ventricular dysfunction were randomized to CABG (499 patients) or CABG + SVR (501 patients). Median age was 62 years with no significant differences in baseline characteristics in both treatment groups despite time of surgery. 30 day as well as 5-year cardiovascular mortality was not different between $\mathrm{CABG}$ and $\mathrm{CABG}+\mathrm{SVR}$. The primary
Table 1 Hazard ratios for cardiovascular events related to LDLcholesterol and hs-CRP levels

\begin{tabular}{llll}
\hline $\begin{array}{l}\text { LDL cholesterol } \\
(\mathrm{mg} / \mathrm{dl}) \text { and hs-CRP } \\
(\mathrm{mg} / \mathrm{l}) \text { levels }\end{array}$ & $\begin{array}{l}\text { Events/ } \\
\text { patients, } n\end{array}$ & $\begin{array}{l}\text { Event } \\
\text { rate }\end{array}$ & $\begin{array}{l}\text { Hazard } \\
\text { ratio }(95 \% \mathrm{CI})\end{array}$ \\
\hline hs-CRP levels $<2 \mathrm{mg} / \mathrm{l}$ & & & \\
$\geq 70$ and $\geq 2$ & $31 / 1384$ & 1.11 & $1.06(0.72-1.55)$ \\
$\geq 70$ and $<2$ & $8 / 726$ & 0.54 & $0.42(0.18-0.94)$ \\
$<70$ and $\geq 2$ & $41 / 2921$ & 0.62 & $0.53(0.38-0.74)$ \\
$<70$ and 2 & $23 / 2685$ & 0.38 & $0.35(0.23-0.54)$ \\
$\geq 70$ or $\geq 2$ & $80 / 5031$ & 0.38 & $0.64(0.49-0.84)$ \\
hs-CRP levels $<1 \mathrm{mg} / 1$ & & & \\
$\geq 70$ and $\geq 1$ & $36 / 1874$ & 0.95 & $0.89(0.62-1.28)$ \\
$\geq 70$ and $<1$ & $3 / 236$ & 0.64 & $0.46(0.11-1.85)$ \\
$<70$ and $\geq 1$ & $59 / 4662$ & 0.56 & $0.49(0.37-0.66)$ \\
$<70$ and $<1$ & $5 / 944$ & 0.24 & $0.21(0.09-0.51)$ \\
$\geq 70$ or $\geq 1$ & $98 / 6772$ & 0.67 & $0.59(0.46-0.75)$ \\
\hline
\end{tabular}

endpoint (death or hospitalization for cardiac causes) was also not different (CABG 58\%, CABG + SVR 59\%, n.s., Fig. 2). In both treatment groups, the CCS, NYHA-classification and the 6-min walking test improved to the same extent despite end-systolic volume index (20 vs. $3 \%$ in CABG alone), which improved in SVR. Thus additional SVR-surgery is not beneficial in patients with ischemic heart failure [6].

\section{FIX-HF-5: multicenter randomized controlled trials of cardiac contractility modulation in patients with advanced heart failure}

Patients with advanced heart failure with a functional NYHA-class III-IV and a EF $\leq 35 \%$ were enrolled in the FIX-HF-5-trial to evaluate the influence of an implanted electrical cardiac contractility modulation (CCM) device on anaerobic threshold and quality of life. The signals of the CCM-device are non-excitatory delivered during the absolute refractory period only to improve cardiac contractility [2]. The safety endpoint of mortality and hospitalization after 12 months was not different in both groups (medical treatment: 213 patients; medical treatment +CCM: 215 patients). Moreover the primary efficacy endpoint (anearobic threshold, AT) could not be improved significantly by CCM compared to medical treatment alone. However, quality of life, NYHA-classification and peak VO2 significantly increased in patients with CCM compared to medical treatment alone. Moreover, a subgroup-analysis revealed a significant benefit regarding the primary endpoint of AT in patients with an EF $\leq 25 \%$, but as a major limitation, there was no blinding in this trial. 


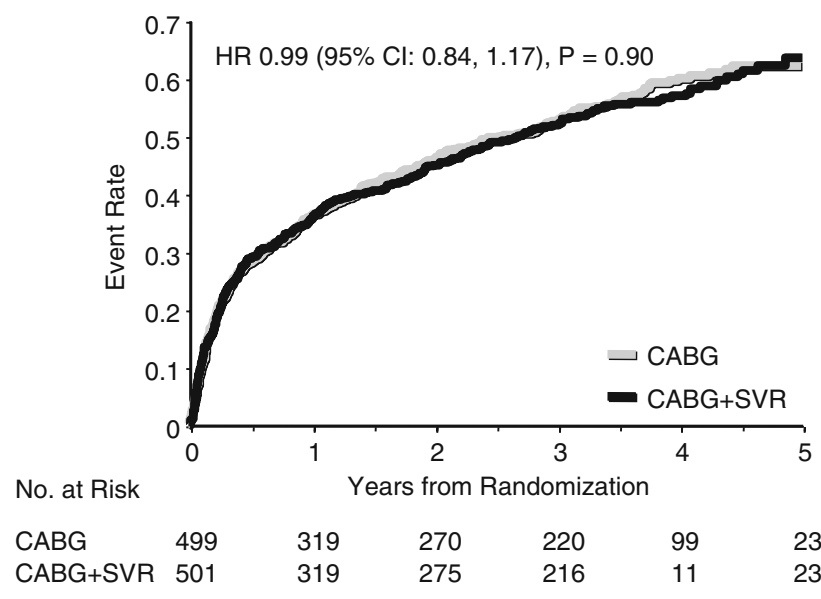

Fig. 2 Kaplan-Meier estimates of the rate of hypothesis 2 primary endpoints of death or cardiac hospitalization

\section{Pre-RELAX-AHF-Study: relaxin, a novel treatment for acute heart failure}

Relaxin is a natural human peptide, mediating vasoregulatory effects especially during pregnancy [9]. In this trials 234 patients with acute heart failure (dyspnoea, congestion on chest radiograph, and increased brain natriuretic peptide (BNP) with a normal blood pressure $(>125 \mathrm{mmHg})$ were assigned to placebo $(n=62)$ or relaxin $10 \mu \mathrm{g} / \mathrm{kg}(n=40)$, $30 \mu \mathrm{g} / \mathrm{kg} \quad(n=43), 100 \mu \mathrm{g} / \mathrm{kg} \quad(n=39)$, or $250 \mu \mathrm{g} / \mathrm{kg}$ $(n=50)$ per day. Mean time of enrollment was $6 \mathrm{~h}$ with a treatment period of $48 \mathrm{~h}$. Blood pressure reduction was not significantly different from placebo in all relaxin treatment groups in patients with a systolic blood pressure below $140 \mathrm{mmHg}$ at baseline. In patients with an initial systolic blood pressure above $140 \mathrm{mmHg}$, there was a significant reduction by relaxin. Clinical endpoints as dyspnea and reduction of edema improved with relaxin $30 \mu \mathrm{g} / \mathrm{kg}$ compared to placebo. Length of hospital stay was reduced in relaxin-treated patients (10.2 \pm 6.1 days) compared to placebo (12.0 \pm 7.3 days). Cardiovascular death and days alive out of hospital were significantly reduced for $30 \mu \mathrm{g} /$ $\mathrm{kg}$ at days 60 and 180. There were no differences of adverse or serious adverse events in all treatment groups. Moreover, renal function was not affected by relaxin treatment. These results demonstrate the safety of relaxintreatment in acute heart failure and are the basis for further clinical trials [12].

\section{PRIMA-study: can pro-brain natriuretic peptide guided therapy of heart failure improve heart failure morbidity and mortality?}

Patients with chronic heart failure were managed with a fixed NT-proBNP level in most trials, but many patients fail to reach this target [8]. The PRIMA-study investigated, whether individually management of chronic heart failure by pro-BNP levels improves morbidity and mortality. Inclusion criteria were admission to hospital with symptomatic heart failure and an increased NT-proBNP of at least $200 \mathrm{pmol} / \mathrm{l}$. The NT-proBNP guided group $(n=174)$ was managed individually with regard to the lowest NT-proBNP level in the first 2 weeks after beginning of treatment of acute HF, whereas the clinical judgement group $(n=171)$ was not. Within a median FU of 702 days, there was no statistical difference either in the primary endpoint (days alive outside hospital, 685 days for BNP group, 664 days for control) or in cardiovascular mortality or hospitalizations. Total mortality was $26.5 \%$ in the BNP-guided patients and $33.3 \%$ in clinically guided patients. However, NT-proBNP guided management resulted in significantly more frequent start or increase of heart failure medications.

HORIZONS-AMI-trial: predictors of stent thrombosis after primary angioplasty in acute myocardial infarction

The new analysis of the HORIZONS-AMI trial evaluated risk factors for late stent thrombosis (up to 1 year) after primary PCI in ST-elevation myocardial infarction in 3,202 patients treated with stenting [11]. Stent thrombosis occurred in $3.3 \%$ of patients $(0.9 \%$ acute, $1.6 \%$ subacute, $1.0 \%$ late). There was no difference of rate of stent thrombosis in patients treated with a drug-eluting or a baremetal stent respectively. Risk factors for late stent thrombosis were insulin treated diabetes, ongoing smoking, high number of stents, treatment of ulcerated lesions and total occlusion of the coronary artery responsible for MI. Moreover, there was no statistically different rate of stentthrombosis in patients receiving bivalirudin $(3.6 \%) \mathrm{com}-$ pared to heparin and GP IIb/IIIa-inhibitor (3.2\%). High-dose clopidogrel $(600 \mathrm{mg})$ was found to be protective, especially for subacute stent-thrombosis.

\section{JUPITER: a randomized trial of rosuvastatin in the prevention of venous thromboembolism}

The use of statins might be beneficial not only in arterial, but also venous disorders. Venous thromboembolism (VTE) was a secondary endpoint in the JUPITER-trial evaluating the effect of rosuvastatin in primary prevention in 17,802 patients [7]. Within a median follow-up of about 2 years, there was a significant reduction of VTEs in the rosuvastatin group (34 events) compared to placebo (60 events, RRR $43 \%, p=0.007$, Fig. 3). This result was consistent after restriction to unprovoked $(n=50)$ and 
provoked events ( $n=44$, cancer, hospitalization, surgery, trauma). $93 \%$ of VTEs occured before a cardiovascular event without a significant difference between estimated relative hazard of VTE (0.57) and primary cardiovascular (0.56) as a first event. The benefit of decreased rates of VTEs was consistent within all subgroups.

\section{Eprotirome as a novel and physiological approach} to CVD risk reduction: beneficial effects on lipoprotein levels when added to statin therapy

Lipid metabolism regulated by thyroid hormones takes place in the liver. Eprotirome is a liver-selective thyroid receptor agonist and was evaluated for safety and lipid-lowering effects combined with a statin in 189 patients with hypercholesterolemia [5]. All lipid markers, especially LDL $(-25 \%)$, triglycerides and lipoprotein(a) (both -30 to $-40 \%)$ were significantly reduced with eprotirome. Nonhigh-density lipoproteins and apolipoprotein B were reduced by $25 \%$. Thus the substance might be beneficial in patients who need further LDL-reduction beyond statin treatment or with a mixed hypercholesterolemia respectively. Within the trials, eprotirome was safe and function restricted to the liver.

Signs of subclinical coronary atherosclerosis measured as coronary artery calcification improve risk prediction of hard events beyond traditional risk factors in an unselected general population: the Heinz Nixdorf recall study 5 -year outcome data

The prospective cohort-study evaluated more than 4,800 patients without known coronary artery disease for pre- dictors and signs of subclinical atherosclerosis. The 5-year outcome data were herein presented with the coronary artery calcium score (measured by electron-beam tomography) being a better predictor of cardiovascular disease compared to classic risk factors. However, the risk prediction was more valid in men than in women and is suggested to improve risk prediction of patients, especially with intermediate risk.

\section{Treatment of intermittent claudication with hypoxia-inducible factor- $1 \alpha$}

Two hundred and eighty-nine patients with claudication and PAD in both legs (67 years, mean ABI 0.6 in the index leg) were randomized to three different doses of hypoxiainducible factor (HIF)- $1 \alpha$ or placebo administered to the thigh and calf muscle. After 3 and 6 months follow-up neither peak walking time, walking distance, claudication onset time nor ABI could be improved. Thus, gene therapy with HIF- $1 \alpha$ offers no benefit in for PAD.

\section{ACTIVE A: clopidogrel plus aspirin versus aspirin alone for prevention of vascular events in patients with atrial fibrillation at high risk of stroke}

In this trial, 7,554 patients with atrial fibrillation and a high risk of stroke for whom vitamin $\mathrm{K}$-antagonist were unsuitable were randomized to aspirin or the combination of aspirin and clopidogrel $(75 \mathrm{mg})$. Median follow-up was 3.6 years with a relative risk reduction of major vascular events in the combination group of $11 \%(p=0.01)$. This result was mainly due to a reduction of stroke (combination
Fig. 3 Cumulative incidence of venous thromboembolism in the JUPITER-trial

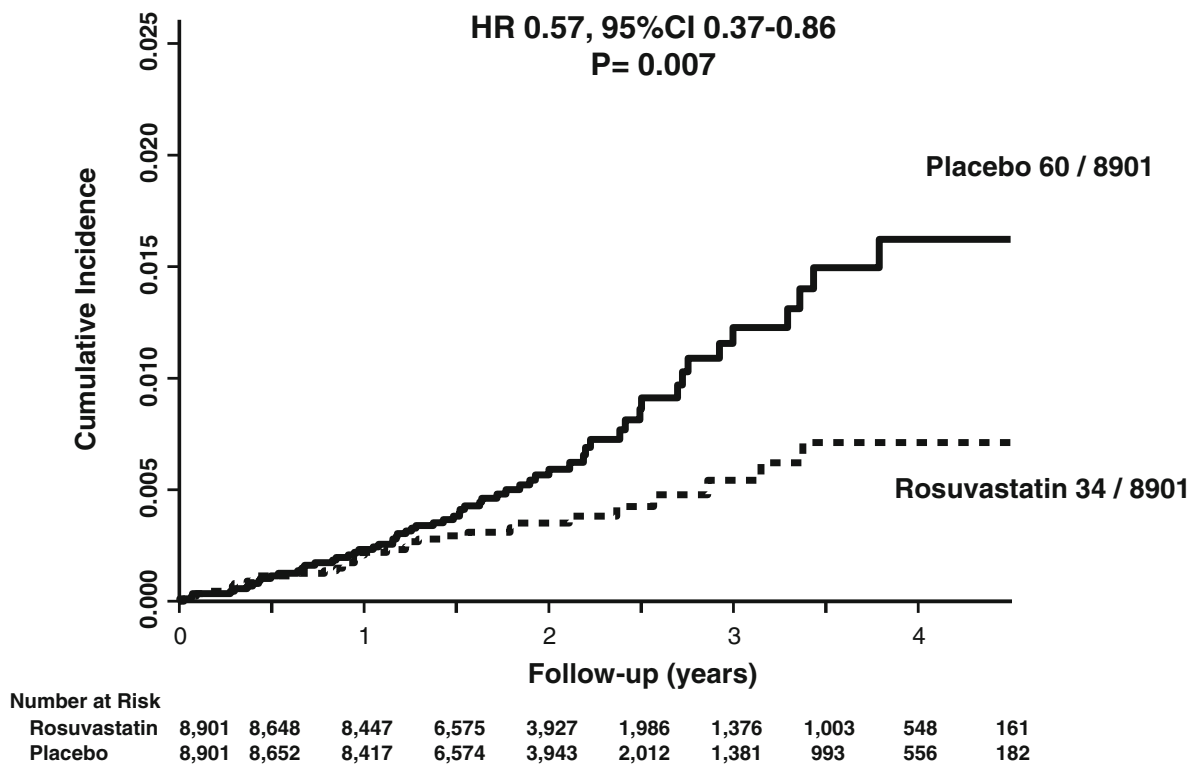


$2.4 \%$ per year vs. aspirin $3.3 \%$ per year, $p<0.001$, Fig. 4 ). Rate of myocardial infarction was not statistically significant different (combination $0.7 \%$ per year vs. aspirin $0.9 \%$ per year, $p=0.08)$. In contrast, major bleeding was more common in the combination treatment group $(2.0 \%$ per year) compared to aspirin alone $(1.3 \%$ per year, $p<0.001)$. Thus, the combination of clopidogrel and aspirin in patients with atrial fibrillation and a high risk of stroke reduces the rate of stroke, but increases major hemorrhage.

\section{Cardiac resynchronization therapy prevents disease progression in NYHA class I-II heart failure patients: 24 months results from the european cohort of the REVERSE trial}

The results of this trial represent the data of the 262 European participants of the REVERSE-trial with 180 patients in the verum-group with enabled CRT function compared to 82 patients with disabled device function. After a follow-up of 24 months, patients with enabled devices had a reduced LVESV (69.7 vs. $94.5 \mathrm{ml} / \mathrm{m}^{2}$, $p<0.0001)$ and LVEDV (103 vs. $132 \mathrm{ml} / \mathrm{m}^{2}, p<0.0001$ ). In contrast to the 12 months follow-up results, clinical worsening was significantly less frequent in CRT-patients (19\%) compared to non-CRT patients $(34 \%, p<0.01)$. Moreover, there was a $62 \%$ RRR in time to first hospitalization or death in patients with enabled CRT-device. However, the primary end-point composite of all-cause mortality, heart-failure hospitalizations, crossover due to worsening heart failure, NYHA class, and the patient global assessment was not significantly different in both treatment groups. This is most likely to the missing change of functional parameters, including the Minnesota Living

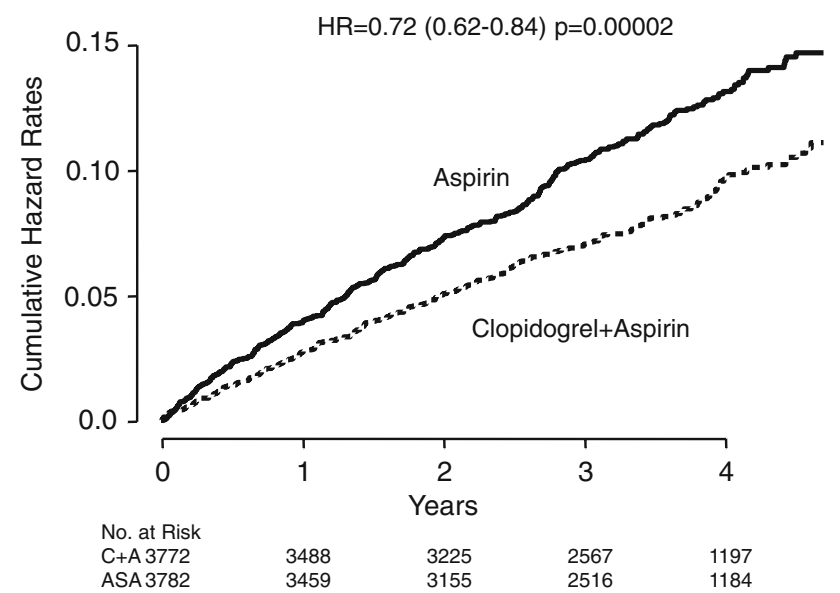

Fig. 4 Influence of combination therapy with clopidogrel + ASS in atrial fibrillation on stroke with Heart Failure Questionnaire, the 6-min walk test, and NYHA class.

\section{ADMIRE-HF-trial: prognostic significance of 123I-mIBG myocardial scintigraphy in heart failure patients}

123I meta-iodobenzylguanidine is a physiologic analogue of norepinephrine, taken up into sympathetic nerves. Due to prior single centre trials, a lower uptake of $123 \mathrm{I}$ mIBG in patients with heart failure is associated with a poor outcome. In this trial, 964 patients with chronic heart failure in a functional NYHA-class II-III were quantified regarding cardiac uptake of the tracer. The heart/mediastinum-ratio (H/M) was determined with a cut-off of 1.6 for a highuptake. After a follow-up for a maximum of 2 years, the composite endpoint of NYHA-class progression, potentially life-threatening arrhythmic event and cardiac death was significantly more frequent in patients with a low uptake $(37 \%)$ of the tracer (high-uptake 15\%). Moreover, there were 51 cardiac deaths in the low-uptake and only two cardiac deaths in the high-uptake group with a negative predictive value of $98.8 \%$ for cardiac death in patients with a high uptake. Thus, myocardial scintigraphy might be a useful tool in risk stratification of patients with chronic heart failure.

\section{References}

1. Indian Polycap Study (TIPS), Yusuf S, Pais P, Afzal R, Xavier D, Teo K, Eikelboom J, Sigamani A, Mohan V, Gupta R, Thomas N (2009) Effects of a polypill (Polycap) on risk factors in middleaged individuals without cardiovascular disease (TIPS): a phase II, double-blind, randomised trial. Lancet 373(9672):1341-1351

2. Abraham WT, Burkhoff D, Nademanee K, Carson P, Bourge R, Ellenbogen KA, Parides M, Kadish A (2008) A randomized controlled trial to evaluate the safety and efficacy of cardiac contractility modulation in patients with systolic heart failure: rationale, design, and baseline patient characteristics. Am Heart J 156(4):641-648e1

3. Fellstrom BC, Jardine AG, Schmieder RE, Holdaas H, Bannister K, Beutler J, Chae DW, Chevaile A, Cobbe SM, GronhagenRiska C, De Lima JJ, Lins R, Mayer G, McMahon AW, Parving HH, Remuzzi G, Samuelsson O, Sonkodi S, Sci D, Suleymanlar G, Tsakiris D, Tesar V, Todorov V, Wiecek A, Wuthrich RP, Gottlow M, Johnsson E, Zannad F (2009) Rosuvastatin and cardiovascular events in patients undergoing hemodialysis. N Engl J Med 360(14):1395-1407

4. Giugliano RP, White JA, Bode C, Armstrong PW, Montalescot G, Lewis BS, van THA, Berdan LG, Lee KL, Strony JT, Hildemann S, Veltri E, Van de Werf F, Braunwald E, Harrington RA, Califf RM, Newby LK. (2009) Early versus delayed, provisional eptifibatide in acute coronary syndromes. N Engl J Med (epub ahead of print)

5. Howard WV (1993) IV or IA lines? Anaesth Intensive Care 21(1):126-127

6. Jones RH, Velazquez EJ, Michler RE, Sopko G, Oh JK, O'Connor CM, Hill JA, Menicanti L, Sadowski Z, Desvigne-Nickens P, 
Rouleau JL, Lee KL (2009) Coronary bypass surgery with or without surgical ventricular reconstruction. $N$ Engl $J$ Med 360(17):1705-1717

7. Mollmann H, Nef H, Bohm M, Laufs U (2009) Highlights of the hotline sessions presented at the scientific sessions 2008 of the American Heart Association. Clin Res Cardiol 98(1):1-7

8. Ndrepepa G, Braun S, Mehilli J, Niemoller K, Schomig A, Kastrati A (2007) A prospective cohort study of prognostic power of $\mathrm{N}$-terminal probrain natriuretic peptide in patients with non-ST segment elevation acute coronary syndromes. Clin Res Cardiol 96(1):30-37

9. Regitz-Zagrosek V, Gohlke-Barwolf C, Geibel-Zehender A, Haass M, Kaemmerer H, Kruck I, Nienaber C (2008) Heart diseases in pregnancy. Clin Res Cardiol 97(9):630-665

10. Rupp H (2006) Omega-3 fatty acids in secondary prevention after myocardial infarct. Clin Res Cardiol 95(Suppl 6):VI12-VI16
11. Stone GW, Witzenbichler B, Guagliumi G, Peruga JZ, Brodie BR, Dudek D, Kornowski R, Hartmann F, Gersh BJ, Pocock SJ, Dangas G, Wong SC, Kirtane AJ, Parise H, Mehran R (2008) Bivalirudin during primary PCI in acute myocardial infarction. N Engl J Med 358(21):2218-2230

12. Teerlink JR, Metra M, Felker GM, Ponikowski P, Voors AA, Weatherley BD, Marmor A, Katz A, Grzybowski J, Unemori E, Teichman SL, Cotter G (2009) Relaxin for the treatment of patients with acute heart failure (Pre-RELAX-AHF): a multicentre, randomised, placebo-controlled, parallel-group, dosefinding phase IIb study. Lancet 373(9673):1429-1439

13. Voller H (2006) Significance of changes in habits followed by risk reduction. Clin Res Cardiol 95(Suppl 6):VI6-VI11 